\title{
RELAPSING POLYCHONDRITIS, AN UNDERESTIMATED ARTICULAR AND DERMATOLOGICAL URGENCY - A CASE REPORT
}

\begin{abstract}
Marco Antonio Gonçalves Pontes Filho1,^, Jhonatan Costa Messias¹, Patrícia Lúcia Silva Sampaio Leite², Victor Gomes Rocha ${ }^{3}$, Mariana Sandy Mada ${ }^{4}$, Pedro Gustavo Barbosa Lira², Vinícius Shinoda Pereira ${ }^{5}$, Natália Fernandes Malfacini², Júlio César Furlan
\end{abstract}

1.Hospital Ipiranga, São Paulo (SP), Brazil; 2.Universidade Federal de Alagoas, Maceió (AL), Brazil; 3.Centro Universitário Tiradentes, Maceió (AL), Brazil; 4.Universidade Federal de Santa Catarina, Florianópolis (SC), Brazil; 5.Universidade Cesumar, Maringá (PR), Brazil.

*Corresponding author: marcoantonio_mcz@yahoo.com.br

\section{BACKGROUND}

Relapsing polychondritis (RP) is a rare multisystemic disease widely accepted as a complex autoimmune disorder affecting proteoglycan-rich structures and cartilaginous tissues, especially the auricular pinna, cartilage of the nose, tracheobronchial tree, eyes, and heart's connective components. Approximately one-third of the cases occur in association with other rheumatologic and hematologic disorders, usually some form of systemic vasculitis, a connective tissue disorder or a myelodysplastic syndrome. This implicates in a high risk of misdiagnosis and contributes to define its complexity at a diagnostic and also therapeutic level. Diagnosis of RP is mainly based on clinical clues, while laboratory data have only a supportive contribution.

\section{CASE REPORT}

A 63-year-old female patient went to the emergency department with a history of bilateral pain, swelling, and redness in the outer ear for a year, associated with polyarthritis of wrists and elbows, and hoarseness of voice and wheezing (Figure 1). There was no trauma, sunburn, or irritant exposure. Her medical history was relevant for well-controlled diabetes, hypothyroidism, knee osteoarthritis, and hypertension. She was prescribed several courses of antibiotics for presumed cellulitis and erysipelas. Despite completing these courses, there was minimal symptom improvement. After one appointment 3 months earlier, she was started on hydroxychloroquine $400 \mathrm{mg} / \mathrm{day}$, also without improvement. On physical examination, the helix was erythematous and exquisitely tender, with the earlobe spared. Her wrists and elbows were swollen. No fever, lymphadenopathy, sinus tenderness, or tympanic membrane abnormalities detected. Results of her cardiopulmonary examination revealed no respiratory findings or heart murmurs, as well as imaging test. The admission laboratory tests showed normal ESR and CRP. Rheumatoid factor, ANA and ANCA were negative. Serologies for HIV, HBC and HCV also negative, and positive IgG for EBV and CMV. Normal joints X-rays. She was then started on prednisone $40 \mathrm{mg} /$ day, with substantially improvement in her discomfort. The clinical diagnosis of RP was made, as there was chondritis at two noncontiguous locations demonstrating a response to corticosteroids. She was eventually transitioned to corticosteroid-sparing therapy of $15 \mathrm{mg}$ of methotrexate weekly successfully.
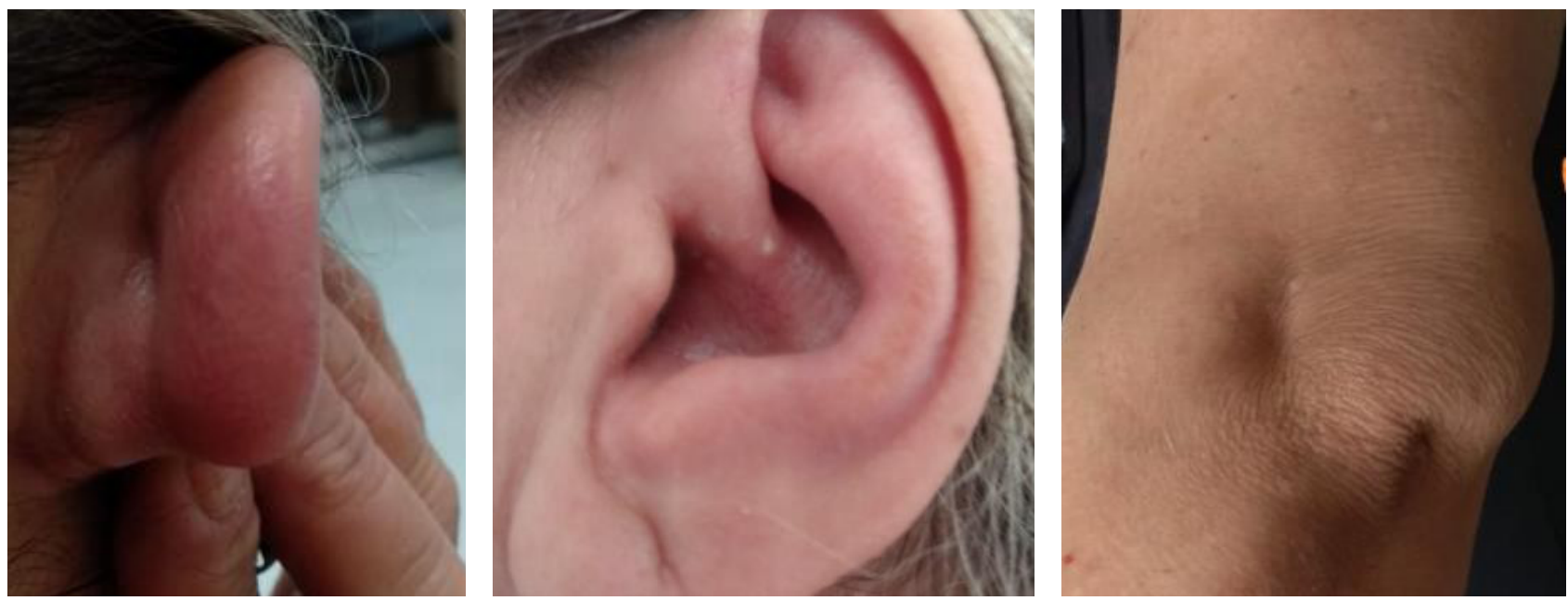

Figura 1. Inflammed ear and elbow.

Realização: 


\section{CONCLUSION}

Relapsing polychondritis is rare disease and presents similarly to other conditions including cellulitis and contact dermatitis. Its epidemiology remains unknown because of the relatively small number of affected patients. A high index of suspicion is needed, particularly when the inflammation is limited to cartilaginous structures. Identification of the classical symptoms is very important in order to obtain the diagnosis, initiate treatment, avoid serious complications of the disease, and manage associated complications.

\section{KEYWORDS}

Relapsing polychondritis, Rare diseases, Differential diagnosis, Cartilage. 